Published in final edited form as:

Transl Cancer Res. 2017 May ; 6(Suppl 3): S512-S514. doi:10.21037/tcr.2017.03.81.

\title{
Bacteriogenic magnetic nanoparticles as magnetic resonance imaging contrast agents
}

\author{
Dawen Zhao ${ }^{1,2}$ \\ ${ }^{1}$ Department of Biomedical Engineering, Wake Forest School of Medicine, Winston Salem, NC, \\ USA
}

${ }^{2}$ Department of Cancer Biology, Wake Forest School of Medicine, Winston Salem, NC, USA

\begin{abstract}
Magnetic resonance imaging (MRI), which has excellent spatial resolution and soft tissue contrast, is a commonly used imaging tool for clinical disease diagnosis. MRI contrast agents are often administered to enhance the image contrast between normal and diseased tissues because of their ability to shorten the relaxation time of the surrounding water molecules. Paramagnetic gadolinium-based metal chelates have predominantly been using as $\mathrm{T}_{1}$ MRI contrast agents in clinic. There is another class of MRI contrast agents, superparamagnetic iron oxides (SPIOs), which, instead of altering $\mathrm{T}_{1}$, have dominant effect on decreasing the transverse relaxation time $\left(\mathrm{T}_{2}\right)(1)$. In contrast to the $\mathrm{T}_{1}$ contrast agents that generate brighter signals, SPIOs produce darker signals, so called negative contrast. However, SPIOs have much higher molar relaxivity and are thus widely used for molecular MRI applications such as cell tracking and molecular targeting (2,3). SPIO nanoparticles that comprise mainly magnetite, the ferric form of iron oxide, can be synthesized with high uniformity in size at varied diameters or lengths.

Intriguingly, some microbes are found to have innate ability to synthesize magnetite to form specific intracellular organelles, the magnetosomes (4). Among these microbes, magnetotactic bacteria (MTB), a group of Gram-negative bacteria have been extensively studied. The MTB magnetosome is composed of a protein-rich lipid bilayer membrane and the enclosed crystals of magnetic iron oxides (5). Individual magnetosomes are aligned in a linear chain by attaching to a cytoskeletal filament, which allow the bacterial to navigate along the geomagnetic field. The size of magnetosomes is highly uniform but varies between species. Genomic analysis of MTBs has identified a number of genes that are highly likely to be involved in regulation of magnetosome biosynthesis. These genes are responsible for encoding various membrane proteins that are essential to either maintain the structural integrity or transport iron, the building blocks of magnetosome $(6,7)$.
\end{abstract}

Attracted by its paramagnetic property, researchers have been exploring the potential of magnetosomes as useful MRI contrast agents (8). Isolation and purification of

Correspondence to: Dawen Zhao, MD, PhD. Department of Biomedical Engineering, Wake Forest School of Medicine, Medical Center Boulevard, Winston-Salem, NC 27157, USA. dawzhao@ wakehealth.edu.

Conflicts of Interest: The author has no conflicts of interest to declare.

Provenance: This is a Guest Editorial commissioned by the Section Editor Xian-Xin Qiu [Shanghai Proton and Heavy Ion Center (SPHIC), a.k.a. the Proton and Heavy Ion Center of Fudan University Shanghai Cancer Center (FUSCC), Shanghai, China]. 
magnetosomes from bacteria have previously been studied in terms of their paramagnetic relaxivity (9). In the March 2017 issue of Biomaterials, Boucher et al. have taken a further step to develop genetically modified magnetosomes with surface expression of RGD peptides, which enables MRI to monitor their specific targeting to av $\beta 3$ integrinsoverexpressing brain tumors in a mouse model of glioma (10). This study utilizes the gene construct of MamC that is known to associate with magnetosome production in Magnetospirillum magneticum AMB-1 strain. Genetic fusion of MamC with the RGD sequence and a yellow fluorescence reporter gene enables the AMB-1 strain to express RGD peptides at the outer surface of magnetosomes. The RGD-magnetosomes purified from the bacterial present a uniform size of $40 \mathrm{~nm}$. The authors conduct both in vitro and in vivo studies showing the RGD-magnetosomes have excellent binding specificity to av $\beta 3$ integrins-expressing tumor cells. Importantly, in vivo $\mathrm{T}_{2}{ }^{*}$-weighted MRI provides both temporal and spatial information of intratumoral biodistribution of the RGD-targeted probe in orthotopic U87 gliomas. As presented in the article, there is initially no difference in intratumoral signal decrease on $\mathrm{T}_{2}$-weighted images between RGD-labeled and non-labeled magnetosomes at earlier time points post systemic administration. However, MRI clearly reveals significantly more RGD-targeted magnetosomes in glioma at $24 \mathrm{~h}$, indicating their ability to bind to av $\beta 3$ integrins-expressing tumor vascular endothelial cells and tumor cells and subsequently become internalized into the cells.

Compared to the chemically synthesized SPIOs, the transverse relaxivity $\mathrm{r}_{2}\left(560 \mathrm{mM}^{-1} \mathrm{~s}^{-1}\right.$ measured at 11T) of the magnetosomes reported in this study is notably higher, which may also contribute to improved MRI sensitivity. As presented in the MR images, SPIO, the $T_{2}$ contrast agent, generates negative contrast on $\mathrm{T}_{2}$ or $\mathrm{T}_{2}{ }$-weighted images. It is noticeable that signal loss due to SPIO shortening of $\mathrm{T}_{2}$ relaxation time is often difficult to differentiate from those low signals induced by B0 inhomogeneity or susceptibility artifacts, occurring particularly with $\mathrm{T}_{2}$-weighted sequences. Longitudinal MRI measurements may provide a solution to the problem by comparing pre and post SPIO administration. To overcome this drawback, various strategies including the off-resonance imaging techniques and the "hotspot" analysis have been exploited, aiming to generate positive contrast of SPIO $(11,12)$. Alternatively, it is recognized that SPIOs also exhibit a high longitudinal relaxivity $r_{1}$, which may be utilized to generate positive $T_{1}$ contrast if applied with appropriate imaging sequences. Indeed, recent studies have shown that a ultrashort echo time (UTE) imaging sequence with very short echo time (TE), typically below $0.1 \mathrm{~ms}$, is able to minimize $\mathrm{T}_{2}$ effect to provide $\mathrm{T}_{1}$-weighted signal enhancement (13).

Even though glioblastoma multiforme (GBM) is composed of highly angiogenic and leaky microvessels, it is well recognized that disruption of blood brain barrier (BBB) in GBM is heterogeneous, indicating many intratumoral regions still contain the intact BBB. Much effort has been made to improve delivery of therapeutic or imaging agents to brain tumors by penetrating the BBB. Although various strategies have been explored to improve drug permeation into brain tumors via physical or chemical means to manipulate the tumor BBB, limited success has been achieved. Integrins, the cell surface adhesion molecules that connect the extracellular matrix (ECM) to the cytoskeleton have been identified to overexpress on neovascular endothelial cells during tumor angiogenesis. In particular, av $\beta 3$ integrin has been a well-recognized angiogenic biomarker $(14,15)$. In addition to its vascular 
expression, av $\beta 3$ integrins are also found to present abundantly on glioma cells. Hence, a number of monoclonal antibodies, peptides, and peptidomimetic agents against av $\beta 3$ integrin have been developed. For example, Cilengitide, a cyclic RGD-based peptide, is being tested for treatment of clinical GBM. Cyclic RGD peptides have also been used to facilitate targeted delivery of imaging contrast agents or anti-cancer therapeutics. In particular, a number of nanocarriers functionalized with surface RGD peptides have been convincingly shown to deliver therapeutic or imaging agents to brain tumor tissues (16).

Alternative to the use of magnetosomes isolated from the bacteria, several studies have introduced the magnetosome gene constructs into the mammalian cell genome (17). For example, magA, another gene identified in Magnetospirillum magneticum AMB-1 strain, which is known for its role on iron transportation, has been transduced into several types of mammalian cells including stem cells $(17,18)$. Like those magnetosome-producing bacteria, these magA containing mammalian cells are able to produce intracellular magnetosomes. As one of the main applications of molecular MRI is to track the cells labeled with SPIOs, the magnetosome gene-transduced stem cells or lymphocytes seem ideal to serve for this purpose. The information obtained by non-invasive in vivo MRI of biodistribution of the cells will be valuable for stem cell therapy or immunotherapy.

Safety index of imaging contrast agents is critical for their in vivo application in preclinical studies and ultimate clinical translation. There are concerns about if introduction of magnetosomes or the magnetosome reporter gene into the mammalian cells may have adverse effects on the cells. As reported in this article and also observed by several other groups, the isolated magnetosomes are biocompatible and thus safe to use at the dose of $\sim 200 \mu \mathrm{mol} / \mathrm{kg}$. However, the authors are still cautious about its possible long term side effect. Given the bacteria-derived products, magnetosomes can trigger the host immunity. Although this is not the case in this work in which the immunocompromised mice are used, further studies in this respect will be necessary. In summary, the work by Boucher and colleagues has established genetically modified bacterial magnetosome with surface expression of tumor-targeting ligands, and successfully demonstrated its potential as a useful brain tumor-targeted imaging contrast agent for molecular MR imaging.

\section{Acknowledegements}

Funding: Brain tumor research has been supported in part by NIH R01 CA194578 and Wells Fargo Scholar Program.

\section{References}

1. Weissleder R Target-specific superparamagnetic MR contrast agents. Magn Reson Med 1991;22:209-12; discussion 213-5. [PubMed: 1812348]

2. Bulte JW, Kraitchman DL. Iron oxide MR contrast agents for molecular and cellular imaging. NMR Biomed 2004;17:484-99. [PubMed: 15526347]

3. Remsen LG, McCormick CI, Roman-Goldstein S, et al. MR of carcinoma-specific monoclonal antibody conjugated to monocrystalline iron oxide nanoparticles: the potential for noninvasive diagnosis. AJNR Am J Neuroradiol 1996;17:411-8. [PubMed: 8881233]

4. Blakemore R Magnetotactic bacteria. Science 1975;190:377-9. [PubMed: 170679] 
5. Uebe R, Schüler D. Magnetosome biogenesis in magnetotactic bacteria. Nat Rev Microbiol 2016;14:621-37. [PubMed: 27620945]

6. Lohsse A, Ullrich S, Katzmann E, et al. Functional analysis of the magnetosome island in Magnetospirillum gryphiswaldense: the mamAB operon is sufficient for magnetite biomineralization. PLoS One 2011;6:e25561. [PubMed: 22043287]

7. Faivre D, Schüler D. Magnetotactic bacteria and magnetosomes. Chem Rev 2008;108:4875-98. [PubMed: 18855486]

8. Goldhawk DE, Rohani R, Sengupta A, et al. Using the magnetosome to model effective gene-based contrast for magnetic resonance imaging. Wiley Interdiscip Rev Nanomed Nanobiotechnol 2012;4:378-88. [PubMed: 22407727]

9. Mériaux S, Boucher M, Marty B, et al. Magnetosomes, biogenic magnetic nanomaterials for brain molecular imaging with 17.2 T MRI scanner. Adv Healthc Mater 2015;4:1076-83. [PubMed: 25676134]

10. Boucher M, Geffroy F, Prévéral S, et al. Genetically tailored magnetosomes used as MRI probe for molecular imaging of brain tumor. Biomaterials 2017;121:167-78. [PubMed: 28088078]

11. Zurkiya $\mathrm{O}, \mathrm{Hu} \mathrm{X}$. Off-resonance saturation as a means of generating contrast with superparamagnetic nanoparticles. Magn Reson Med 2006;56:726-32. [PubMed: 16941618]

12. Zhou H, Stafford JH, Hallac RR, et al. Phosphatidylserine-targeted molecular imaging of tumor vasculature by magnetic resonance imaging. J Biomed Nanotechnol 2014;10:846-55. [PubMed: 24734537]

13. Wang L, Zhong X, Qian W, et al. Ultrashort echo time (UTE) imaging of receptor targeted magnetic iron oxide nanoparticles in mouse tumor models. J Magn Reson Imaging 2014;40:107181. [PubMed: 25485347]

14. Hood JD, Cheresh DA. Role of integrins in cell invasion and migration. Nat Rev Cancer 2002;2:91-100. [PubMed: 12635172]

15. Xiong JP, Stehle T, Zhang R, et al. Crystal structure of the extracellular segment of integrin alpha Vbeta3 in complex with an Arg-Gly-Asp ligand. Science 2002;296:151-5. [PubMed: 11884718]

16. Luo Y, Yang J, Yan Y, et al. RGD-functionalized ultrasmall iron oxide nanoparticles for targeted T1-weighted MR imaging of gliomas. Nanoscale 2015;7:14538-46. [PubMed: 26260703]

17. Zurkiya O, Chan AW, Hu X. MagA is sufficient for producing magnetic nanoparticles in mammalian cells, making it an MRI reporter. Magn Reson Med 2008;59:1225-31. [PubMed: 18506784]

18. Pereira SM, Williams SR, Murray P, et al. MS-1 magA: Revisiting Its Efficacy as a Reporter Gene for MRI. Mol Imaging 2016;15 pii: 1536012116641533. [PubMed: 27118760] 